\title{
A Pre-clinical Rat Model for the Study of Ischemia- reperfusion Injury in Reconstructive Microsurgery
}

\author{
Alberto Ballestín ${ }^{1}$, Javier G. Casado ${ }^{2}$, Elena Abellán ${ }^{1}$, Francisco Javier Vela ${ }^{1}$, José Luis Campos ${ }^{1}$, Guadalupe Martínez- \\ Chacón ${ }^{1}$, Jorge Bote ${ }^{3}$, Rebeca Blázquez ${ }^{2}$, Francisco Miguel Sánchez-Margallo ${ }^{1,2,3}$ \\ ${ }^{1}$ Department of Microsurgery, Jesús Usón Minimally Invasive Surgery Centre ${ }^{2}$ Stem Cell Therapy Unit, Jesús Usón Minimally Invasive Surgery \\ Centre ${ }^{3}$ Animal Modelling Unit, Jesús Usón Minimally Invasive Surgery Centre
}

\section{Corresponding Author}

Alberto Ballestín

balles_rodriguez@hotmail.com

\section{Citation}

Ballestín, A., Casado, J.G., Abellán, E., Vela, F.J., Campos, J.L., MartínezChacón, G., Bote, J., Blázquez, R., Sánchez-Margallo, F.M. A Pre-clinical Rat Model for the Study of Ischemiareperfusion Injury in Reconstructive Microsurgery. J. Vis. Exp. (153), e60292, doi:10.3791/60292 (2019).

\section{Date Published}

November 8, 2019

DOI

$10.3791 / 60292$

URL

jove.com/video/60292

\section{Abstract}

Ischemia-reperfusion injury is the main cause of flap failure in reconstructive microsurgery. The rat is the preferred preclinical animal model in many areas of biomedical research due to its cost-effectiveness and its translation to humans. This protocol describes a method to create a preclinical free skin flap model in rats with ischemia-reperfusion injury. The described $3 \mathrm{~cm} \times 6 \mathrm{~cm}$ rat free skin flap model is easily obtained after the placement of several vascular ligatures and the section of the vascular pedicle. Then, $8 \mathrm{~h}$ after the ischemic insult and completion of the microsurgical anastomosis, the free skin flap develops the tissue damage. These ischemia-reperfusion injury-related damages can be studied in this model, making it a suitable model for evaluating therapeutic agents to address this pathophysiological process. Furthermore, two main monitoring techniques are described in the protocol for the assessment of this animal model: transit-time ultrasound technology and laser speckle contrast analysis.

\section{Introduction}

Microsurgery has become a common surgical technique for reconstruction that allows for interventions (e.g., free tissue transfers) to restore complex tissue defects, replantation of amputated limbs, and even composite tissue allotransplantations.

Microsurgical reconstructions are ideal for a wide variety of defects caused by traumatic injuries, burns, or oncological resections. However, there is a low percentage of free flap failure, among which ischemia-reperfusion (I/R) injury is one of the main responsible factors. All microsurgically transferred tissues endure a mandatory period of ischemia followed by the reperfusion. This period of primary ischemia is usually well tolerated; thus, the success rate of microsurgical procedures exceeds $90 \%{ }^{1,2}$. However, only $63.7 \%$ of 
flaps requiring surgical revision can be completely saved ${ }^{3}$. In addition, in cases of replantation of finger avulsion injuries, the success rate is $66 \%{ }^{4}$; and in cases of composite tissue allotransplantation suffering $I / R$ injury, rejection percentages are increased since the $I / R$ injury activates innate immunity 5,6 .

Therefore, the study of this pathophysiological phenomenon is of interest. Animal models are essential for investigating physiological mechanisms and assessing novel therapies before it can be applied to humans ${ }^{7}$. Vessel anatomy and the physiological similarities between rats and human beings make rats an ideal model for the investigation of biological processes such as I/R injury.

Here, we present a detailed protocol for the creation of a rat free skin flap model with I/R injury, as well as different possibilities for intra- and postoperative assessments. The overall goal of this method is to describe a useful preclinical model to study I/R injury and possible treatments to reduce its related damages.

\section{Protocol}

All procedures were conducted in accordance with the ethical committee of the Jesús Usón Minimally Invasive Surgery Centre and the welfare guidelines of the regional government that are based on European legislation.

\section{Presurgical and Surgical Preparation}

1. House Wistar rats weighing $290-350 \mathrm{~g}$ in cages at 22$25{ }^{\circ} \mathrm{C}$ with free access to food and water. Acclimate for 1 week prior to the surgery to prevent stress-induced problems.
2. Place a rat in an anesthetic induction chamber, deliver 5 min of oxygen $(0.5-1 \mathrm{~L} / \mathrm{min})$, and use a vaporizer to deliver $5 \%$ sevoflurane to induce anesthesia.

3. Take the rat out of the chamber once anesthesia is induced. Place the inhalation facemask on the rat and provide a flow rate of $2 \%$ sevoflurane to maintain anesthesia. Check for the lack of response to a toe pinch.

4. Use an eye protection ointment to prevent corneal drying and damage.

5. Monitor the animal under general anesthesia as follows: Place a rectal thermometer $\left(35.9-37.5^{\circ} \mathrm{C}\right)$, check the mucous membrane color, and position a rodent pulse oximeter to check for the $\mathrm{O}_{2}$ saturation ( $>95 \%$ ) and heart rate $(250-450 \mathrm{bpm})$.

6. Use a heat support (electric heating pads or circulating water blankets) to avoid hypothermia and improve postprocedural anesthesia recovery.

7. Inject $5 \mathrm{~mL}$ of warm subcutaneous physiological saline solution to maintain proper hydration.

8. Provide analgesic and anti-inflammatory drugs (meloxicam $1 \mathrm{mg} / \mathrm{kg} /$ day) and prophylactic antibiotics (enrofloxacin $7.5 \mathrm{mg} / \mathrm{kg} /$ day) subcutaneously before the procedure and for 5 days postoperatively.

9. Shave the animal's abdominal and inguinal areas.

10. Apply topical povidone-iodine, followed by $70 \%$ ethanol. Cover the animal with a sterile drape.

\section{Free Skin Flap Model Surgery}

1. Using a surgical marker, draw a $3 \mathrm{~cm} \times 6 \mathrm{~cm}$ flap matching one of the $6 \mathrm{~cm}$ sides with the abdomen midline. Next, make a $6 \mathrm{~cm}$ skin incision at the midline 
of the abdomen and two perpendicular $3 \mathrm{~cm}$ incisions at the upper and lower part of the $6 \mathrm{~cm}$ midline incision.

2. To start dissecting the designed $3 \mathrm{~cm} \times 6 \mathrm{~cm}$ skin flap, use scissors and Adson forceps to raise the flap (rather than a scalpel) due to the skin's mobility.

3. Gently pull the flap from the cranial area towards the caudal area to help with the dissection and identify the epigastric pedicle surrounded by the abundant loose connective tissue.

4. Dissect the flap pedicle without touching it or by grasping the adventitia as little as possible to avoid damaging the vessel wall.

5. Use $8 / 0$ nylon sutures to occlude by ligatures the proximal caudal femoral vessels, lateral circumflex femoral vessels, and saphenous vessels. Thereby, the perfusion of the flap is provided by the femoral artery and continues directly through the superficial caudal epigastric artery, while the venous drainage is performed by the superficial caudal epigastric vein toward the femoral vein.

6. Clamp the vascular pedicle and then cut it to start the $8 \mathrm{~h}$ ischemia period. During the procedure use electric blankets to maintain temperature. Two $5 \mathrm{ml}$ injections of warm $\left(25^{\circ} \mathrm{C}\right) 0.9 \%$ saline solution are administered subcutaneously. The first administration is performed 2 $\mathrm{h}$ after the beginning of the procedure; and the second at the end of the procedure to obtain a proper recovery of the animal.

7. Use heparinized saline solution $(100 \mathrm{U} / \mathrm{mL})$ to perfuse the flap and remove the stagnant blood from the microcirculation.

8. Use $10 / 0$ nylon sutures to perform the microsurgical anastomoses.
9. After $8 \mathrm{~h}$ of ischemia, reperfuse the flap by removing the microvascular clamps and check the vascular patency as described below.

\section{Intraoperative Assessment}

1. Perform a manual patency test (empty and refill test) for the vein and artery. To do this, use two microsurgical forceps, position them distal to the anastomosis and perform the milking. Release the closest forceps to the anastomosis site first. If the blood flow continues after a vascular section is emptied, then the anastomosis is patent.

2. Assess the blood flow using a transit-time ultrasound flowmeter and microsurgical probes.

1. Measure the diameter of the pedicle vessels in order to choose the proper size for the flow probes.

NOTE: A $0.7 \mathrm{~mm}$ flow probe can measure vessels ranging from $0.4 \mathrm{~mm}$ to $0.7 \mathrm{~mm}$; a $1.0 \mathrm{~mm}$ flow probe can measure vessels ranging from $0.7 \mathrm{~mm}$ to $1.0 \mathrm{~mm}$; a $1.5 \mathrm{~mm}$ flow probe can measure vessels ranging from $1.0 \mathrm{~mm}$ to $1.5 \mathrm{~mm}$.

2. Place the target vessel in the ultrasonic sensing window (between the reflector and the transducers) of the flow probe to quantify flow volume.

NOTE: Hold the probe neutral to the plane of the vessel, to avoid any tension or pulling.

3. Check the quality of the acoustic coupling by observing that all the bars are green on the display. NOTE: If difficult to get good acoustic coupling, use ultrasonic gel or physiological saline solution topically.

4. When good coupling is achieved and the vessel is placed in the acoustic window without any tension, 
click on the Record button on the display to store the data.

NOTE: To obtain a reliable and correct measurement, make sure that the waveform pattern is constantly repeatable.

3. Once done, use Polyglycolic Acid (PGA) 4-0 absorbable braided sutures (16mm 3/8 triangular needle) to close the skin. Use a simple interrupted pattern to maintain strength and tissue position if part of the suture is bitten off by the rat postoperatively.

4. Assess the flap's microcirculation using laser speckle contrast analysis (LASCA).

1. Make a new recording for each animal and for each follow-up of the study. For this, click File/New Recording. A new window opens, and the Setup Panel is displayed. Then edit the information of the project name, subject, operator and recording name.

2. For maximum reproducibility, standardize the following parameters: working distance, measurement area, point density, frame rate, and ambient light conditions.

1. Adjust the Working Distance by moving the laser in relation to the tissue. Zoom in or out the laser head towards the tissue of interest. To check for the measured value, click Image Setup. Here, set at $12.0 \mathrm{~cm}$.

2. Standardize the Measurement Area by entering the desired Width and Height at the Image Setup. The designed flap measures 3 $\mathrm{cm} \times 6 \mathrm{~cm}$. For this measurement, select a width of $4.0 \mathrm{~cm}$ and a height of $7.0 \mathrm{~cm}$ to have some extra space.
3. Set the Point Density as high in the Image Setup. High, medium, and low are the three options.

3. At the Image Capture Setup, select the Frame Rate (10 images/s) for the recording and the Duration (1 $\mathrm{min}$ ) of the recording.

NOTE: Have the same ambient light condition in the surgery room while operating or performing the assessments.

4. Click the Record button to start recording. The Setup Panel is replaced by the Recording Panel. Data is saved automatically. Take snapshots during the procedure to enable further comparison.

NOTE: The perfusion scale can be changed to improve visualization (Click Tools | Filters and Color Scales | Perfusion Scale | Manual 0 150), but the measured perfusion values will be unaffected. Before and after the recording, different regions of interest (ROIs) can be created to measure the perfusion within them. Here, we evaluated just the area of the practiced flap $(3 \mathrm{~cm} \times 6 \mathrm{~cm})$.

5. Use ImageJ software to measure the survival and necrosis areas.

1. Locate a ruler at the side of the flap and then take control pictures for macroscopic measurements of the flap survival area.

2. To evaluate pictures, open the ImageJ user interface. Click File and Open the image to be measured.

3. Select Straight Line at the toolbox and draw a straight line over $1 \mathrm{~cm}$ of the ruler. Click on Analyze I Set Scale and introduce in the text box for known distance the value of $1 \mathrm{~cm}$. 
4. Click on the Polygon Selection Tool and draw the polygon lines over the flap to calculate the viable area. Ultimately, click on Analyze | Measure to obtain the area value.

6. Place a postoperative dressing on the animal before housing to prevent self-mutilation of the surgical area. After the procedures, animals are housed in cages individually, in a room with temperature control $\left(22^{\circ} \mathrm{C}\right.$ to $\left.25^{\circ} \mathrm{C}\right)$.

\section{Postoperative Assessment and Tissue Sampling}

1. Anesthetize the rat at 7 postoperative days for the flap assessment and tissue sampling by following the same steps previously described in this protocol (steps 1.2 and 1.3). Check for the depth of anesthesia by the lack of response to the toe pinch.

2. Photograph the surgical area to enable macroscopic measurements of the flap survival and necrosis areas. Make the postoperative macroscopic measurements following the same steps of the intraoperative assessment that have been explained before in the protocol (step 3.5).

NOTE: Pay attention while using the Polygon Selection Tool by drawing the lines on the flap delimiting the viable area (measured in $\mathrm{cm}^{2}$ ). The percentage of the viable area can be calculated as $\left(\mathrm{cm}^{2}\right.$ of viable area/ $\mathrm{cm}^{2}$ of total flap area) $\times 100$.
3. Assess the flap's microcirculation using the LASCA technique (step 3.4 ) to visualize and quantify perfusion differences

4. After the macroscopic analysis, remove the $4 / 0$ sutures and rise the flap to reassess the vascular pedicle blood flow by using the transit-time ultrasound.

5. Perform tissue sampling by longitudinally dividing the flap into two parts of $1.5 \mathrm{~cm} \mathrm{x} 6 \mathrm{~cm}$.

1. Immerse one part in a biopsy container with $4 \%$ paraformaldehyde at room temperature for further histological analyses.

2. Introduce the other part of the tissue in a cryopreservation tube, immerse it in liquid nitrogen and then, cryopreserve the tube by storing it at -80 ${ }^{\circ} \mathrm{C}$ for future molecular analyses.

6. Euthanize the rat under general inhalation anesthesia using a rapid intracardiac injection of $2 \mathrm{M} \mathrm{KCl} / \mathrm{kg}$ according to the ethical committee recommendations.

\section{Representative Results}

Immediately after the creation of the microsurgical anastomoses, we obtained higher blood flow volumes than the minimum flows recommended in the literature ${ }^{8}$; thus, all microsurgical anastomoses were patent 1 week after the surgery (Figure 1). 

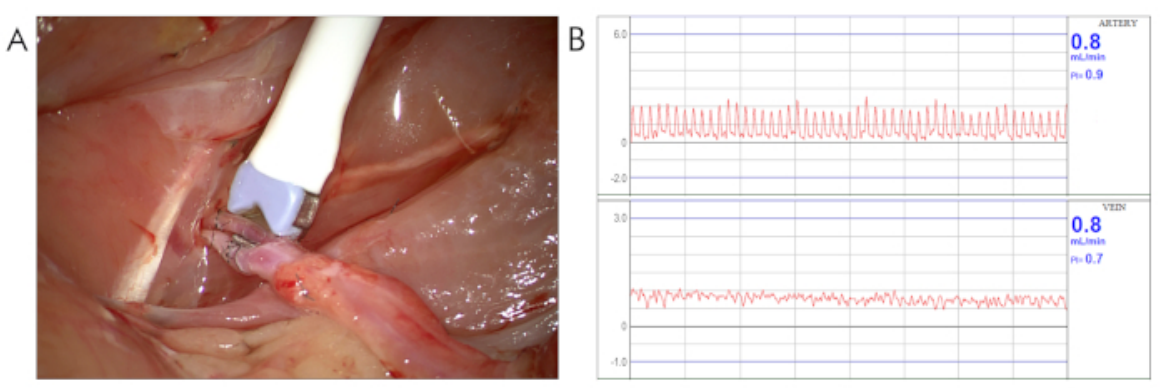

Figure 1: Transit-time ultrasound blood flow assessment. (A) Position of the microsurgical flow probe to assess blood flow. (B) Blood flow pattern and quantification obtained of the anastomosed vessels of the flap pedicle. Please click here to view a larger version of this figure.

Observation of the microcirculatory deprivation of blood flow during the ischemic insult was possible with the LASCA technique, including the immediate hyper perfusion during the flap reperfusion, and, perioperatively, the different areas with less perfusion and a higher risk of postoperative flap necrosis that were indeed necrotized 7 days after the end of the study (Figure 2).
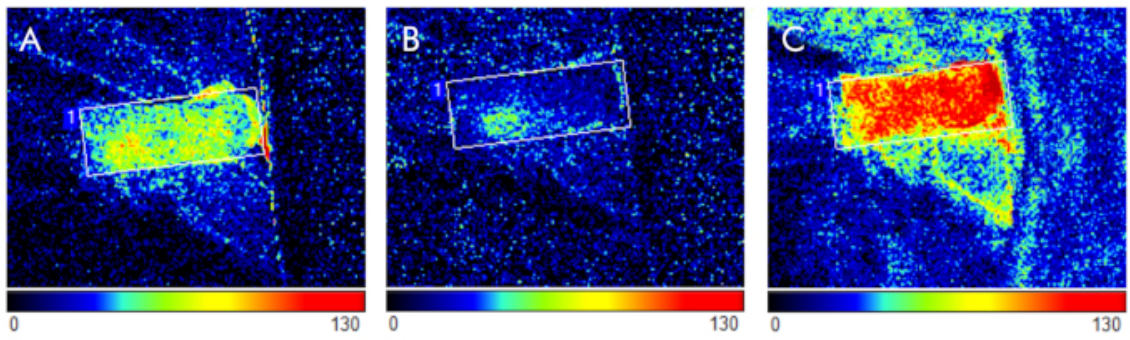

Figure 2: Laser speckle contrast analysis technology. (A) Visualization of microcirculatory tissue blood perfusion in the physiological condition. (B) Visualization of microcirculatory tissue blood perfusion during ischemia. (C) Visualization of microcirculatory tissue blood perfusion immediately after reperfusion. Please click here to view a larger version of this figure.

The flap survival area after $8 \mathrm{~h}$ of ischemia and its subsequent reperfusion was around $40 \%$. Previously published results ${ }^{9}$ showed statistically significant differences when this model was compared with flaps where no ischemic insult was inflicted.

\section{Discussion}

Microsurgical free tissue transfers have become the method of choice for reconstructing large defects. A period of ischemia occurs during such free tissue transfers. When this period exceeds the tissue's tolerance, I/R injury can cause 
failure of the practiced free flap ${ }^{9}$. The description of the methodology to develop a cost-effective and translational preclinical model to study $\mathrm{I} / \mathrm{R}$ injury in reconstructive microsurgery may help lead the study of different compounds to counteract this pathophysiological process.

In the described animal model, after the vascular ligatures were placed and the free flap was raised, no hindlimb blood flow compromises were noted, nor pain or limp. As Kochi et al. ${ }^{10}$ described, our model also left three collateral routes through intramuscular networks.

Monitoring of free flaps is of major importance ${ }^{11}$, as salvage is inversely related to the duration between ischemia onset and its clinical recognition. For this purpose, free flaps should be studied intra- and postoperatively.

Intraoperatively, the widely used empty and refill test or the acoustic Doppler enable identification but not quantification of flow presence or absence through an anastomosis ${ }^{12}$. For this reason, we used transit-time ultrasound technology, a novel method that allows surgeons to quantify the blood flow of microsurgical anastomoses ${ }^{13}$. In our study, all microsurgical anastomoses were patent after $8 \mathrm{~h}$ of ischemic insult as well as at the end of the study. Immediately after the creation of the microsurgical anastomoses, we noted higher blood flow volumes than the minimums recommended in literature ${ }^{8}$. This predicted good pedicle perfusion at the end of the study, demonstrating that the results were not influenced by the microsurgical technique but rather by the $\mathrm{I} / \mathrm{R}$ injury cascade of events. However, this technique is not free of limitations. To obtain reliable results, the microsurgical probes must be held neutral to the plane of the vessel, not pulling it or creating any tension. A good acoustic coupling is needed to obtain a proper signal, which can be achieved using ultrasonic gel or saline. A high-quality coupling signal, provided by the equipment, is an important parameter to consider during the measurements.

We have used LASCA, also known as laser speckle contrast imaging or laser speckle imaging, postoperatively ${ }^{14}$. This technology represents a valuable technique for semiquantitative real-time mapping of flow within free flaps as verified here. One of the limitations is that the results are provided in arbitrary units and not directly related to actual flow values. In this sense, further research is needed to validate this correlation. Laser Doppler flowmetry is more commonly used but limited by the fact that it only measures perfusion in a single point in the flap, whereas LASCA allows the detection of regional changes in skin perfusion within the flap ${ }^{15}$. Furthermore, a recent study ${ }^{16}$ indicated that LASCA may perioperatively predict the regions at high risk of postoperative flap necrosis. Our results suggest that LASCA is a promising technique for the peri- and postoperative monitoring of free flaps.

\section{Disclosures}

The authors have nothing to disclose.

\section{Acknowledgments}

The research project was performed at the Jesús Usón Minimally Invasive Surgery Center (CCMIJU), part of the ICTS Nanbiosis. The study was performed with assistance from the following Nanbiosis units: U21, experimental operating room; U22, animal housing; and U14, cell therapy. This work was supported by ISCIII project PI16/02164 and by European Regional Development Fund (FEDER), a way to make Europe. The funder had no role in the study design, data collection and analysis, decision to publish, or manuscript preparation. Special thanks are extended to María Pérez for preparing the figures and to Fernanda Carrizosa 
for providing constant encouragement and supporting the scientific bibliography.

\section{References}

1. Siemionow, M., Arslan, E. Ischemia/reperfusion injury: a review in relation to free tissue transfers. Microsurgery. 24 (6), 468-475 (2004).

2. Wang, W. Z., Baynosa, R. C., Zamboni, W. A. Update on ischemia-reperfusion injury for the plastic surgeon: 2011. Plastic and Reconstructive Surgery. 128 (6), 685e-692e (2011).

3. Chen, K. T. et al. Timing of presentation of the first signs of vascular compromise dictates the salvage outcome of free flap transfers. Plastic and Reconstructive Surgery. 120 (1), 187-195 (2007).

4. Sears, E. D., Chung, K. C. Replantation of finger avulsion injuries: a systematic review of survival and functional outcomes. The Journal of Hand Surgery. 36 (4), 686-694 (2011).

5. Caterson, E. J., Lopez, J., Medina, M., Pomahac, B., Tullius, S. G. Ischemia-reperfusion injury in vascularized composite allotransplantation. The Journal of Craniofacial Surgery. 24 (1), 51-56 (2013).

6. Amin, K. R., Wong, J. K. F., Fildes, J. E. Strategies to Reduce Ischemia Reperfusion Injury in Vascularized Composite Allotransplantation of the Limb. The Journal of Hand Surgery. 42 (12), 1019-1024 (2017).

7. Barre-Sinoussi, F., Montagutelli, X. Animal models are essential to biological research: issues and perspectives. Future Science OA. 1 (4), FSO63 (2015).

8. Shaughness, G., Blackburn, C., Ballestin, A., Akelina, Y., Ascherman, J. A. Predicting Thrombosis Formation in 1-mm-Diameter Arterial Anastomoses with TransitTime Ultrasound Technology. Plastic and Reconstructive Surgery. 139 (6), 1400-1405 (2017).

9. Ballestin, A. et al. Ischemia-reperfusion injury in a rat microvascular skin free flap model: A histological, genetic, and blood flow study. PloS One. 13 (12), e0209624 (2018).

10. Kochi, T. et al. Characterization of the Arterial Anatomy of the Murine Hindlimb: Functional Role in the Design and Understanding of Ischemia Models. PloS One. 8 (12), (2013).

11. Smit, J. M., Zeebregts, C. J., Acosta, R., Werker, P. M. Advancements in free flap monitoring in the last decade: a critical review. Plastic and Reconstructive Surgery. 125 (1), 177-185 (2010).

12. Krag, C., Holck, S. The value of the patency test in microvascular anastomosis: Correlation between observed patency and size of intraluminal thrombus: An experimental study in rats. British Journal of Plastic Surgery. 34 64-66, (1981).

13. Selber, J. C. et al. A prospective study of transit-time flow volume measurement for intraoperative evaluation and optimization of free flaps. Plastic and Reconstructive Surgery. 131 (2), 270-281 (2013).

14. Briers, D. et al. Laser speckle contrast imaging: theoretical and practical limitations. Journal of Biomedical Optics. 18 (6), 066018 (2013).

15. Zotterman, J., Bergkvist, M., Iredahl, F., Tesselaar, E., Farnebo, S. Monitoring of partial and full venous outflow obstruction in a porcine flap model using laser speckle contrast imaging. Journal of Plastic, Recontructive and Aesthetic Surgery. 69 (7), 936-943 (2016). 
16. Zotterman, J., Tesselaar, E., Farnebo, S. The use of laser speckle contrast imaging to predict flap necrosis: An experimental study in a porcine flap model. JJournal of Plastic, Recontructive and Aesthetic Surgery. 72 (5), 771-777 (2019). 